\title{
Entropic Order Quantity (EnOQ) Model for Decaying Items with Partial Backordering and Lost Sale
}

\author{
Anima Bag \\ C.V. Raman Group of Institutions Janla, \\ Bhubaneswar, India-752054. \\ P.K. Tripathy \\ P.G. Department of Statistics Utkal University, \\ Vanivihar, Bhubaneswar. \\ Monalisha Pattnaik \\ Dept. of Business Administration, \\ Sambalpur University Burla, Sambalpur, India-768019.
}

\begin{abstract}
In this paper the pricing and lot sizing entropic model for a decaying item under finite production, exponential decay, partial backordering and lost sale is considered. These cost parameters are not easy to eliminate in practice. A new model is framed by introducing new approach of entropy cost. When the customers seem to be impatient, the backlogging option is used. The objective of this model is to maximize the average profit so as to determine the optimal selling price, cycle time for positive inventory, cycle time for stock-out condition and order quantity respectively. The numerical analysis shows that an appropriate policy can benefit the retailer and the policy is important, especially for a decaying item with consideration of entropy. Finally comparative analysis is also done to draw managerial implications for gaining competitive advantage in business functions.
\end{abstract}

AMS subject classification: 90B05.

Keywords: Entropy, EnOQ, Decaying item, Pricing, Partial backlogging. 


\section{Introduction}

As markets have become more and more competitive, disorder has become a prevailing characteristics of modern production system that are operating in complex, dynamic and uncertain environment, Minimizing disorder in these production systems requires stringent control measure by management with cost that are usually hidden or difficult to estimate.

The EOQ model is also known as lot (batch) size problem.It is the oldest scientific approach, and perhaps the simplest one to analyze inventory system. Perishable goods are an important part of stocks carried in practice. The problem of managing inventory of perishable product is complex for a variety of reasons. The product can be usually produced by the vender only at a finite rate. The demand for the product is sensitive to price changed by the vendor to the consumer. Besides carrying cost, the deterioration cost needs to be taken into account. Also, when goods are highly perishable, the vendor may need to back-log demand in order to meet the demands.

In the introspective approach to model backlogging of demand, the inventory manager is required to specify backorder cost and lost sale cost.The difficulty in estimating these costs is well recognized in the inventory literature.In this paper we use Abad (1996)'s model that is suitably redesigned to the backlogging phenomenon. In this approach consumers are considered to be impatient. Hence, when stockout situation occurs, only a fraction of demand occurring at a given time is back ordered. The fraction, moreover is a decreasing function of the waiting time because the approach is revenue based. It is particularly suited to situations in which demand is sensitive to the price charged by manufacturer.

There is considerable literature on problem of determining the lot size of perishable goods under considerations of finite production, fixed demand and backordering. An excellent survey of results in this area is extended by Raafat (1991). Most of the work formulates the problem as cost minimization problem, back order and lost sale costs. Mishra (1975) assumed a Weibull deterioration rate for perishable product without allowing for backlogging of demand. He approximated the total cost per period using Taylor's series approximation. Elsayed et al. (1983) assumed a Weibull deterioration rate for complete backlogging of demands.

Heng et al. (1991) also assumed a constant deterioration rate but used Taylor series approximation, of the exponential and logarithmic expressions in the model and prepared another pattern search procedure. Wee (1991) used Weibull deterioration rate and considers the pricing problems assuming that supplier offers discounts. Wee(1993) allowed demand to be partially backordered.Goyal and Gunasekharan (1995) introduced an integrated production inventory marketing model for deteriorating items with finite replenishment rate. Luo (1998) studied an integrated inventory system for perishable goods with backordering. Recently Abad (2000) has proposed the cost minimization problem in its original form and the global minimum can be obtained using a standard nonlinear programming software without resorting to Taylorseries approximation. Jaber et al. (2008) developed Entropic order Quantity (EnOQ) model for deteriorating items.As markets have more and more competitive, the disorders have become a prevailing charac- 
teristic of modern production systems that are operating in complex environment. Some researchers in management science have applied entropy approaches to account for disorder when modeling the behavior of production systems. They introduced the concept of entropy cost to account for hidden cost such as additional management cost that is needed to control the improvement process.

In classical economic order quantity model, it is often assumed that shortages are either completely backlogged or completely lost. As a physical phenomenon, some customers may likely to prefer backlogging during shortage period, while the other would not. Goyal et al. established an EPQ model for deteriorating items with complete backlogging. In this paper we discuss the inventory policy for the production of entropic lot-sizing model for perishable items with finite production and extend it to infinite planning horizon under partial backlogging and the goal is to determine optimal price as well as lot-size using optimization procedure.

\section{Vendor's Problem}

The assumption in the model are:

1. Planning horizon is infinite.

2. Production rate, which is finite, is more than demand rate.

3. Exponential rate of decay.

4. Demand is represented by a general function i.e the demand function can be any twice differentiable function of price.

5. When Net stockout condition exist, demand is partially backlogged.

\subsection{Notations:}

Let $I(t)$ : Net stock (on hand-back orders) level at time $t$.

$R$ : Production rate for the items (units/period).

$P$ : Selling price within the inventory cycle.

$D(\mathrm{P})$ : demand rate (units/period). $R>D(P)$

$\sigma$ : decay coefficient assumed to be constant. (i.e exponential decay)

$\sigma I(t)$ : Decay rate at time $t$.

$T$ : Duration of inventory cycle when there is positive inventory.

$\lambda$ :Duration of inventory cycle when stockout condition exists.

$\beta, \psi$ : interim time-spans

\subsection{Mathematical Model}

From Figure 1, we can study the nature of the present inventory model when shortage is allowed. We will use $\mathrm{D}$ and $\mathrm{D}(\mathrm{P})$ interchangeably. The following assumptions are made concerning the demand function. 


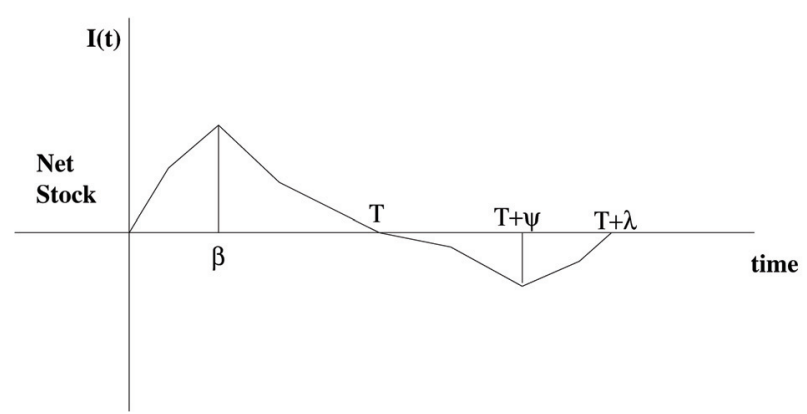

Figure 1: Inventory Model with Shortage

(i) $D^{\prime}=\frac{d D(P)}{d P}<0$ for all $\mathrm{P} \in(0, \infty)$

(ii) The marginal revenue $\frac{d(D(P))}{d D}=P+\frac{D(P)}{D^{\prime}}$ is a strictly increasing function of $\mathrm{P}$ and thus $\frac{1}{D(P)}$ is a convex function of $P$.

When $t \in[0, \beta)$

$$
\frac{d I(t)}{d t}+\sigma I(t)=R-D, R>D, I(0)=0
$$

Similarly when $t \in[\beta, T)$

$$
\frac{d I(t)}{d t}+\sigma I(t)=-D, I(0)=0
$$

Equation (2.1) and (2.2) are linear differential equations.

The solution of differential equation (2.1) is

$$
I(t)=\frac{R-D}{\sigma}\left(1-e^{-\sigma t}\right), t \in[0, \beta)
$$

Similarly the solution of differential equation (2.2) is,

$$
I(t)=\frac{D}{\sigma}\left[e^{\sigma(T-t)}-1\right], t \in[\beta, T)
$$

When $t=\beta$, from equation (2.3) and equation (2.4) we have

$$
I(\beta)=\frac{R-D}{\sigma}\left[1-e^{-\sigma \beta}\right]
$$

and

$$
\begin{gathered}
I(\beta)=\frac{D}{\sigma}\left(e^{\sigma(T-\beta)}\right)-1 \\
\therefore \frac{R-D}{\sigma}\left(1-e^{-\sigma \beta}\right)=\frac{D}{\sigma}\left(e^{\sigma(T-\beta)}-1\right)
\end{gathered}
$$




$$
\begin{gathered}
\Rightarrow R=(R-D) e^{-\sigma \beta}+D e^{\sigma(T-\beta)} \\
=e^{-\sigma \beta}\left[R-D+D e^{\sigma T}\right]
\end{gathered}
$$

Taking logarithm on both sides we get

$$
\begin{aligned}
\ln R & =\ln e^{-\sigma \beta}\left[R-D+D e^{\sigma T}\right] \\
& =\ln e^{-\sigma \beta}+\ln \left[R-D+D e^{\sigma T}\right] \\
& =-\sigma \beta+\ln \left[R-D+D e^{\sigma T}\right] \\
\Rightarrow \sigma \beta & =\ln \left[R-D+D e^{\sigma T}\right]-\ln R \\
& =\ln \frac{R-D+D e^{\sigma T}}{R} \\
\Rightarrow \beta & =\frac{1}{\sigma} \ln \left[\frac{R-D+D e^{\sigma T}}{R}\right]
\end{aligned}
$$

or

$$
\beta=\beta(P, T)=\frac{1}{\sigma} \ln \left[\frac{R-D+D e^{\sigma T}}{R}\right]
$$

\subsection{Backlogging of Demand}

In the above model at a given time $t \in[T, T+\psi]$ shortage occur and only a fraction of demand, $B(\tau)$ is backlogged, where $\tau$ is the amount of time the customer waits before receiving the goods. $B(\tau)$ is a decreasing function of $\tau$ i.e customers are impatient. We use an exponential function (Abad, 1996) to model backlogged demand.

Let

$$
B(\tau)=k_{0} e^{-k_{1} \tau}, k_{0}<1, k_{1} \geq 0
$$

We assume that the customers are served on first come first served basis. At any time $t \in[T, T+\lambda], \tau$ would depend upon the back orders i.e $-I(t))$ because all previous back orders are to be filled first before filling the new back orders. In the fig. 1 during time span $[T, T+\psi]$ there is no production but only backlogging of demands. Thus for $t \in[T, T+\psi]$ the waiting time is given by $\tau=T+\psi-t-\frac{I(t)}{R}$. In time span $[T+\psi, T+\lambda]$ the current backlog is depleted at a rate $\mathrm{R}$ but at the same time new back orders are brought. Hence for $t \in[T+\psi, T+\lambda]$ the waiting time is given by

$$
\tau=\frac{-I(t)}{R}
$$

From above discussion we have for $t \in[T, T+\psi]$

$$
\frac{d I}{d t}=-D B\left(T+\psi-t-\frac{I(t)}{R}\right)=-D k_{0} e^{-k_{1}}\left(T+\psi-t-\frac{I(t)}{R}\right), I(T)=0
$$

The solution to (2.9) for $t \in[T, T+\psi]$ is

$$
I(t)=\frac{R}{k_{1}}\left(\ln \left[\frac{R}{D k_{0} e^{k_{1}(t-T-\psi)}+R-D k_{0} e^{-k_{1} \psi}}\right]\right)
$$


Note

$$
\left.I_{(} \psi\right)=I(T+\psi)=\frac{R}{k_{1}}\left(\ln \left[\frac{R}{D k_{0}+R-D k_{0} e^{-k_{1} \psi}}\right]\right)
$$

Similarly for $t \in[T+\psi, T+\lambda]$

$$
\frac{d I}{d t}=R-D B \frac{-I(t)}{R}=R-D k_{0} e^{-k_{1}\left(\frac{-I(t)}{R}\right)}
$$

Given initial condition (2.11), the solution of differential equation (2.12) for $t \in$ $[T+\psi, T+\lambda]$ is

$$
I(t)=\frac{R}{k_{1}}\left(\ln \left[\frac{R}{R e^{-k_{1}(t-T-\psi)}-D k_{0} e^{-k_{1}(t-T)}+D k_{0}}\right]\right)
$$

From fig. 1, $I(T+\lambda)=0$. Hence letting $t=T+\lambda$ in equation (2.13) and setting $I(T+\lambda)=0$ and solving for $\psi$ we have,

$$
\psi=\psi(P, \lambda)=\frac{1}{k_{1}}\left(\ln \left[\frac{D k_{0}+e^{k_{1} \lambda}\left(R-D k_{0}\right)}{R}\right]\right)
$$

Note that the production occurs in continuous time spans $[0, \beta]$ and $[T+\psi, T+\lambda]$. Hence lot size in this problem is given by $Q=R . \beta+R .(\lambda-\psi)$

$$
Q=R \cdot(\beta+\lambda-\psi)
$$

\subsection{Objective Function}

Let $\mathrm{V}=$ Unit cost

$\mathrm{K}=$ Set up cost

$\mathrm{h}=$ inventory carrying cost /unit/ period

Suppose there is no salvage value for deteriorating items. Then during the inventory cycle of time span $[T+\lambda]$ :,

Revenue $=\mathrm{PDT}+\mathrm{PR}(\lambda-\psi)$

Production cost $V Q=V R \beta+V R(\lambda-\psi)$

Inventory carrying cost $=h \int_{0}^{T} I(t) d t$

$=h \int_{0}^{\beta} \frac{R-D}{\sigma}\left(1-e^{-\sigma t}\right) d t+h \int_{\beta}^{T} \frac{D}{\sigma}\left(e^{\sigma(T-t)}-1\right) d t$

$$
=\frac{h R \beta}{\sigma}+\frac{h(R-D)}{\sigma}\left(e^{-\sigma \beta}-1\right)+\frac{h D}{\sigma}\left(e^{\sigma(T-\beta)}-1\right)-\frac{h D T}{\sigma}
$$

Using (2.5), (2.16) becomes

$$
h \int_{0}^{T} I(t) d t=\frac{h R \beta}{\sigma}-\frac{h D T}{\sigma}
$$




\section{Deterioration Cost:}

We assume that there is no salvage value associated with deteriorated items. The number of deteriorated units is $(\mathrm{P} \beta$ - DT) units. The cost of deterioration, which is incorporated in the cost of production is

$$
V(R \beta-D T)
$$

\section{Entropy Cost:}

The entropy cost per cycle is given by

$\mathrm{EC}=$ Entropy Cost with deterioration

$$
=(D \delta(T))=\frac{Q}{\int_{0}^{T} \frac{D}{P}}=\frac{Q \times P}{D \times T}
$$

Profit during time-span $(0, T+\lambda)$ :

Profit during time span $(0, T+\lambda)$ is

Revenue cost- Inventory carrying cost- Deteriorating cost- Production cost

- Setup cost-Entropy cost

i.e.

$\left.F(P, T, \lambda)=\left(P+\frac{h}{\sigma}\right) D T+R(P-V)[\lambda-\psi(P, \lambda)]-\left(V+\frac{h}{\sigma}\right) \times R \beta(P, T)\right)-K-\frac{Q \times P}{D \times T}$

\section{Average Profit Per Period}

The average profit per period is

$$
\begin{aligned}
\pi(P, T, \lambda) & =\frac{F(P, T, \lambda)}{T+\lambda} \\
& =\frac{\left.\left(P+\frac{h}{\sigma}\right) D T+R(P-V)[\lambda-\psi(P, \lambda))\right]-\left(V+\frac{h}{\sigma}\right) \times R \beta(P, T)-K-\frac{Q \times P}{D \times T}}{T+\lambda}
\end{aligned}
$$

where $\psi(P, T, \lambda)$ is given by expression (2.14) and $\beta(P, T)$ by expression (2.7). The problem faced by vendor is

$$
\begin{gathered}
\left(P_{1}\right) \operatorname{Max} \pi(P, T, \lambda) \\
T \geq 0) \\
\lambda \geq 0) \\
P \geq V)
\end{gathered}
$$

\section{Optimization problem when $P$ is pre-set}

Let selling price $\mathrm{P}$ is assumed to be fixed, profit over time span $T+\lambda$ given in (2.19) will be denoted as $F(T, \lambda, P)$ and objective function will be denoted as $\pi(T, \lambda, P)$.Demand will be denoted as $\mathrm{D}$. The optimization problem is

$$
\left(P_{2}\right) \operatorname{Max} \pi(T, \lambda, P)=\frac{F(T, \lambda, P)}{T+\lambda}
$$




$$
\begin{gathered}
=\frac{\left.\left(P+\frac{h}{\sigma}\right) D T+R(P-V)[\lambda-\psi(P, \lambda))\right]-\left(V+\frac{h}{\sigma}\right) \times R \beta(P, T)-K-\frac{Q \times P}{D \times T}}{T+\lambda} \\
T \geq 0) \\
\psi \geq 0)
\end{gathered}
$$

\subsection{Solution procedure for problem $\mathbf{P 2}$}

Assumption 3.1. The set $G=\{T, \lambda / T+\lambda>0, F(T, \lambda / P)>0\}$ is not a null set.

Proposition 3.2. $F(T, \lambda / P)$ is strictly concave function for $T \geq 0, \lambda \geq 0$.

Proposition 3.3. $\pi(T, \lambda / P)$ is strictly pseudo concave function on $G=\{T, \lambda / T+\lambda>$ $0, F(T, \lambda / P)>0\}$.

\section{Optimization problem when selling price $P$ is a decision variable}

In the previous section a procedure is outlined for determining optimal $\mathrm{T}$ and $\lambda$ when $\mathrm{P}$ is specified. When $\mathrm{P}$ is a decision variable, $F(P, T, \lambda)$ as defined in (2.19) is not a concave function and therefore $\pi(P, T, \lambda)$ as defined in (2.20) cannot be proven to be a pseudo-concave function. For this reason, (P1) can have multiple local maxima. Let $F(P \mid T, \lambda)$ represent the value of $F(P, T, \lambda)$ as defined in (2.19) and let $\pi(P \mid T, \lambda)$ represent $\pi(P, T, \lambda)$ as defined in (2.20) when $T$ and $\lambda$ are fixed. Then from (2.20) $\pi(P / T, \lambda)$ is proportional to $F(P / T, \lambda)$. However convexity of $F(P, T, \lambda)$ is difficult to prove given the complex nature of $\psi(P, \lambda)$ and $\beta(P, T)$. On the other hand, the problem of maximizing $F(P, T, \lambda)$ can be maximized locally using standard non-linear programming software i.e starting with current solution, improvement can be obtained in $F(P, T, \lambda)$ in each iteration. The following procedure makes use of the above.

\subsection{Solution procedure}

1. Let $P=P s$ where $P s$ is some initial value of $P$

2. For current $\mathrm{P}$, solve $\left(P_{2}\right)$ and let the optimal solution to $\left(P_{2}\right)$ be denoted as $T_{p}^{*}, \lambda_{p}^{*}$

3. Let $T=T_{p}^{*}$ and $\lambda=\lambda_{p}^{*}$ and maximize $F(P, T, \lambda)$ locally. Let the value of $\mathrm{P}$ that maximizes $F(P / T, \lambda)$ be the current value of $\mathrm{P}$.

Given proposition 2 , when $\left(P_{2}\right)$ is solved for current $P, \pi(P, T, \lambda)$ should improve. Similarly, $\pi(P, T, \lambda)$ should be improved in step-3, Repeating step-2 and 3 should return a local maximum of $\pi(P, T, \lambda)$. Since there could be multiple local maxima, the above procedure should be repeated with different values of $P_{S}$ to identify the global maximum. Note however that only a starting value in one-dimensional space $(P, T, \lambda)$ is required. The search for global maximum would not be time consuming and can be carried out using standard non-linear programming software. 


\subsection{Numerical Example}

Suppose $D(P)=16,0000 P^{-2}, R=1000$ units/week, $V=$ Rs $10 /$ units, $K=$ $1000 /$ production run, $h=\$ 1.0 /$ unit $/$ week. $\sigma=0.3, K_{0}=0.9, K_{1}=0.6$.

To solve the non linear equations we take the help of the numerical computational software LINGO 13 version, and found optimal solution for EnOQ model. The solution is found to be $P=20.15250, T=1.254833, \lambda=0.6252635, \beta=0.5519521$ and $\psi=0.429322$.Also $\pi(\beta+\lambda-\psi)=2771.149$ units. In this case maximum inventory level $I(\beta)=\frac{D}{\sigma}\left(e^{\sigma(T-\beta)}-1\right)=308.2739021$ units and number of units deteriorated is $\theta=R \beta-D \stackrel{\sigma}{T}=157.983039$ units.

With help of lingo we also found optimal solution for EOQ model. The solution was found to be $P=20.21354, T=1.235266, \lambda=0.6191482, \beta=0.5394279$ and $\psi=0.4261269$. Also $\pi(\beta+\lambda-\psi)=2787.508$ units. In this case maximum inventory level $I(\beta)=\frac{D}{\sigma}\left(e^{\sigma(T-\beta)}-1\right)=303.0131642$ units and number of units deteriorated is $\theta=R \beta-D \stackrel{\sigma}{T}=55.70604008$ units.

Table 1: Optimal Solution of EnOQ and EOQ Models

\begin{tabular}{|l|l|l|l|l|l|l|l|l|l|}
\hline Model & $\mathrm{P}$ & $\mathrm{T}$ & $\lambda$ & $\beta$ & $\psi$ & $I(\beta)$ & $\theta$ & $\mathrm{Q}$ & $\pi$ \\
\hline EnOQ & 20.15250 & 1.254833 & 0.6252635 & 0.5519521 & 0.429322 & 308.2739021 & 157.9830339 & 747.8936 & 2771.149 \\
& & & & & & & & & \\
\hline EOQ & 20.21354 & 1.235266 & 0.6191482 & 0.5394279 & 0.4261269 & 303.0131642 & 55.70604008 & 732.4492 & 2787.508 \\
\hline
\end{tabular}

With introduction to Entropy cost it is found that the selling price $(\mathrm{P})$ within inventory cycle in EnOQ model is slightly less than that of EOQ model. Duration of time when there is positive inventory $(\mathrm{T})$, duration of inventory cycle when stock out condition exists, interim time span $(\beta, \psi)$, maximum inventory level $I(\beta)$, number of units deteriorated $(\theta)$ are more than that of EOQ model. As a result the average profit per period is not much more affected. It is slightly less than that of EOQ model. So the new approach Entropy can be implemented in the model to maximize the average profit.

From managerial point of view, the solution represents the best trade off among pricing and relevant costs which include carrying cost, deterioration cost, entropic cost, back ordering cost and lostsale cost.

\section{Conclusion}

In this paper the pricing and lot sizing entropic model for a decaying item under finite production, exponential decay, partial back ordering and lost sale is considered. These cost parameters are not easy to eliminate in practice. A new model is framed with introducing new approach of entropy cost. When the customers seem to be impatient, the backlogging option is used. The objective of this model is to maximize the average profit so as to determine the optimal selling price, cycle time for positive inventory, cycle time for stock-out condition and order quantity respectively. The numerical analysis 
shows that an appropriate policy can benefit the retailer and the policy is important, especially for a decaying item with consideration of entropy which gives approximately less profit from EOQ model. Finally comparative analysis of EnOQ and EOQ models is also studied to draw managerial implications. This paper may be extended by introducing total backlogging, time value of money, uncertainty, stock and price dependent demand.

\section{References}

[1] Abad, P.L., 1996, "Optimal pricing and lot sizing under conditions of perishability and partial back ordering". Management science 42, 1093-1104.

[2] Abad, P.L., 2000, "Optimal lot size for a perishable good under condition of finite production and partial back ordering and lost sale". Computers and Industrial Engineering 38, 457-465.

[3] Abad, P.L., 2003, "Optimal pricing and lot-sizing under conditions of perishability, finite production and partial back ordering and lot sale". European Journal of Operation Research 144, 677-685.

[4] Bazarra, M. Sheralli H, Shetty, C.M., 1993, "Nonlinear programming”, Second Wiley, New York.

[5] Elsayed, E Teresi, C., 1983, "Analysis of inventory system with deteriorating items". International Journal of production Research 21, 449-460.

[6] Goyal, S.K. Gunasekharan, A., 1995, "An integrated production-inventorymarketing model for deteriorating items with finite replenishment rate". Computer and Industrial Engineering 28, 755-762.

[7] Heng, K.J., Labban, J., Linn, R.J., 1991, "An order level lot size inventory model for deteriorating items with finite replenishment rate", Computer and Industrial Engineering 20, 187-197

[8] Jaber M.Y., Bonney M., Rosen M.A., Monalek I., 2008, "Entropic order quantity (EnOQ) model for deteriorating items". Applied mathematical modelling.

[9] Luo, W., 1998, "An integrated inventory system for perishable goods with backordering". Computers and Industrial Engineering 34, 685-693.

[10] Mishra, R.B., 1975, "Optimum production lot size model for system with deteriorating inventory". International Journal of production Research 13, 495-505.

[11] Raafat, F., 1991, "Survey of literature on continuously deteriorating inventory models". Journal of Operation Research Society 42, 27-37.

[12] Raafat, F. Walfe, P. Eldin, H., 1991, "An inventory model for deteriorating items". Computers and Industrial Engineering 20, 89-94.

[13] Wee, H.M., 1993, "Economic production lot size model for deteriorating items with partial back-ordering". Computers and Industrial Engineering 24, 449-458.

[14] Wee, H.M., 1999, "Deteriorating inventory model with quantity discount, pricing and partial back ordering”. International Journal of Economics 59, 511-518. 